\title{
Measuring nanomechanical motion with a microwave cavity interferometer
}

\author{
C. A. Regal * J. D. Teufel, and K. W. Lehnert \\ JILA, National Institute of Standards and Technology and the University of Colorado, \\ and Department of Physics University of Colorado, Boulder, Colorado 80309, USA
}

(Dated: April 6, 2008)

\begin{abstract}
In recent years microfabricated microwave cavities have been extremely successful in a wide variety of detector applications. In this article we focus this technology on the challenge of quantum-limited displacement detection of a macroscopic object. We measure the displacement of a nanomechanical beam by capacitively coupling its position to the resonant frequency of a superconducting transmission-line microwave cavity. With our device we realize near state-of-the-art mechanical force sensitivity $(3 \mathrm{aN} / \sqrt{\mathrm{Hz}})$ and thus add to only a handful of techniques able to measure thermomechanical motion at 10's of milliKelvin temperatures. Our measurement imprecision reaches a promising 30 times the expected imprecision at the standard quantum limit, and we quantify our ability to extract measurement backaction from our results as well as elucidate the important steps that will be required to progress towards the full quantum limit with this new detector.
\end{abstract}

The advent of micro and nanomechanical resonators has brought the long-standing goal of exploring quantum effects such as superposition and entanglement of macroscopic objects closer to reality. With this ability one could experimentally study decoherence of superposition states thus elucidating questions about the interface between the quantum and classical worlds. Micro and nanomechanical resonators have hastened progress towards macroscopic quantum limits by providing high-frequency, small-dissipation, yet lowmass resonators. Still it remains a challenge to freeze out the thermomechanical motion of these objects to leave only zeropoint fluctuations $\delta x_{z p}$ and, equally importantly, to detect motion at this level. The problem of detection at the quantum limit is in itself intriguing. As the imprecision of any detector is decreased, measurement backaction emerges to enforce the Heisenberg constraint, which for continuous displacement detection is $S_{x}^{i m} S_{F}^{b a} \geq \hbar^{2}$. Here $S_{x}^{i m}$ and $S_{F}^{b a}$ are respectively the displacement imprecision and backaction force spectral densities. In fact, at the minimum allowed total position uncertainty, referred to as the standard quantum limit (SQL), the measurement imprecision and backaction must together contribute an uncertainty equal to the zero-point fluctuations.

A widely used displacement detector that, in principle, is capable of reaching the SQL is an optical cavity interferometer with a moving mirror [1]. Physically, at the SQL ones knowledge of the mirror position is limited equally by shot noise in the output signal and by motion of the mirror due to quantum fluctuations in the intracavity radiation pressure. This limit has long been of interest in quantum optics and in the gravitational wave detection community. Optical cavities generally outperform all other displacement detectors with regard to measurement imprecision; they can achieve shot-noise limited position sensitivity as low as $\sim 10^{-19} \mathrm{~m} / \sqrt{\mathrm{Hz}}$ [2]. Still reaching the SQL has historically been a challenge due to the inaccessibility of quantum backaction effects [3, 4]. Recent experimental progress using low-mass mirrored microcantilevers has made radiation pressure effects more observable $[5,6,6,7,8,9]$.

However, the most successful approaches to the SQL to date have been electromechanical experiments. These exper- iments take place "on-chip" in a dilution refrigerator where thermomechanical motion is significantly reduced, and the mechanical objects are typically nanoscale and hence even less massive than microcantilevers. Examples include using a single electron transistor [10, 11] or an atomic point contact [12] for the displacement readout of a nanoscale flexural beam. Electromechanical experiments have observed a displacement uncertainty less than 10 times the total uncertainty added by the measurement at the SQL and evidence for backaction [10, 11]. Still electromechanical experiments have not achieved the full quantum limit typically due to technical noise sources common to mesoscopic amplifiers.

In this article we present experiments that use the principles and advantages of an optical cavity interferometer with a moving mirror yet employ "light" at microwave frequencies. Operating at microwave frequencies allows us to also benefit from technology associated with electromechanical systems, such as low-mass mechanical objects and dilution refrigerator temperatures. Specifically, we embed a nanomechanical flexural resonator inside a superconducting transmission-line microwave cavity, where the mechanical resonator's position couples to the cavity capacitance and thus to the cavity resonance frequency. Changes in this frequency can be sensitively monitored via homodyne detection of the phase shift of a microwave probe signal. Advantages of superconducting transmission-line cavities include large demonstrated quality factors $\left(Q>10^{5}\right)[13]$ and a tiny mode volume. Additionally the cavities are fabricated via a single deposition of a thin, superconducting film and thus are scalable as well as compatible with patterning of other nanoscale devices. These advantages have been leveraged in an array of other recent applications including microwave kinetic inductance detectors (MKIDS) [13], achieving circuit QED [14], and readout of superconducting quantum interference devices (SQUIDS) [15].

The analogy between our microwave system and an optical cavity interferometer is quite rigorous; the Hamiltonian describing both systems is

$$
H=\hbar \omega_{0}\left(a^{\dagger} a+\frac{1}{2}\right)+\hbar \omega_{m}\left(b^{\dagger} b+\frac{1}{2}\right)-\hbar g a^{\dagger} a\left(b^{\dagger}+b\right) \delta x_{z p}
$$

where the cavity and mechanical modes are described respec- 


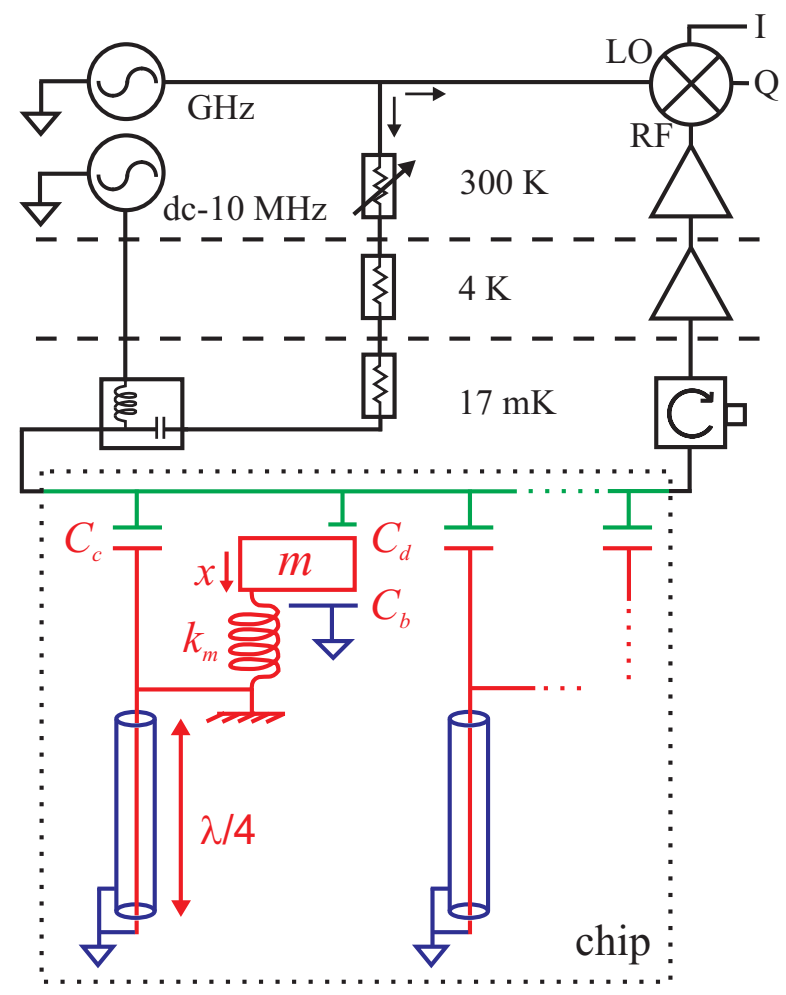

FIG. 1: Measurement schematic. Distributed microwave resonators (red and blue) with a line impedance of $Z_{1}=70 \Omega$ are capacitively coupled, $C_{c}$, to a feedline (green). A nanomechanical beam (red) is coupled to each cavity via a capacitance $C_{b}$ and to the feedline via $C_{d}$ used for an electrostatic drive. The cavity coupling is characterized by $170 \mathrm{aF} / \mu \mathrm{m}$ and the drive coupling by $0.2 \mathrm{aF} / \mu \mathrm{m}$. The beam motion is detected by measuring the phase shift of an injected microwave signal. This signal travels through the device and is then amplified first by a low-noise microwave HEMT amplifier and further at room temperature before going to the rf port of an IQ mixer.

tively by the operators $a$ and $b, \omega_{0}$ and $\omega_{m}$ are the bare resonant cavity and mechanical frequencies, and $g$ is the effect of the displacement $\hat{x}=\left(b^{\dagger}+b\right) \delta x_{z p}$ on the perturbed cavity resonant frequency, $\omega_{c}$. In both cases the Heisenberg limit is enforced, as discussed above, by fluctuations in the optical or microwave field, i.e. shot noise. Still there are important practical differences between optical experiments and our microwave work. While the optical shot-noise limit is achieved routinely, due to the smaller photon energy of microwaves, measurement of microwave fields is currently dominated by amplifier noise. Nonetheless, microwave amplifier technology is progressing quickly, and in our experiments we use a commercially available HEMT amplifier that already reaches a noise temperature of $k_{b} T_{N} / \hbar \omega_{c}=30\left(T_{N}=7.5\right.$ $\mathrm{K})$. The small microwave photon energy also requires an excellent force sensitivity to detect quantum backaction, but our experiments aim to accommodate this requirement by going to the extremes of force sensitivity using floppy mechanical objects.

Our superconducting microwave cavities are formed from
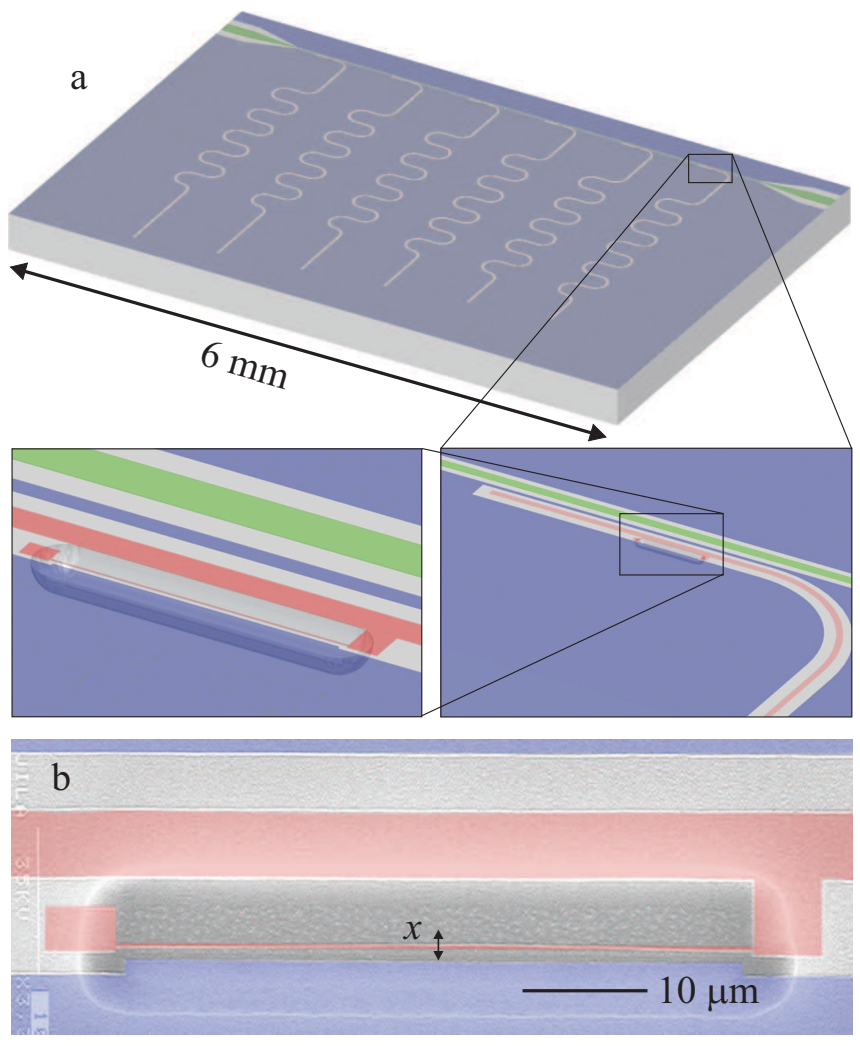

FIG. 2: (a) Drawing of our device showing frequency multiplexed $\lambda / 4$ microwave cavities; the lines are meandered to fit a quarter wave on the chip. The cavity lines are formed from $5 \mu \mathrm{m}$ wide center conductors separated from the ground plane by $10 \mu \mathrm{m}$ slots. The lower panels zoom into a capacitive elbow coupler and a nanomechanical beam with the feedline shown in green, the ground plane in blue, and the center conductor in pink. (b) False color scanning electron microscope image of an embedded nanomechanical beam. This room temperature image shows a top view of the beam, which is clamped on both ends and slightly bent due to compressive stress (see Methods). An angled view of the same beam reveals that it is also bent out of the plane at its center by $2.5 \mu \mathrm{m}$ at room temperature. The area where the silicon was etched to release the beam appears as the darker oblong region.

distributed transmission lines in a coplanar waveguide geometry and are patterned using an aluminum thin film on a highpurity silicon substrate. We use one-sided cavities that are shorted on one end and coupled to a $50 \Omega$ feedline on the other end (Fig. 1 and Fig. 2 a) ); the cavity is overcoupled (dominated by external coupling not by internal losses) to minimize microwave power dissipation. We study multiple cavities on a single chip by coupling six cavities to the feedline and address them individually via frequency multiplexing [13]. The quarter wave resonances of our cavities are near $\omega_{c}=2 \pi \times 5$ $\mathrm{GHz}$.

The nanomechanical objects we embed in the cavity are thin, high aspect ratio beams of conducting $\mathrm{Al}$ clamped on both ends (see Methods). We use a beam $50 \mu \mathrm{m}$ long with a $100 \mathrm{~nm}$ by $130 \mathrm{~nm}$ cross-section (Fig. 2(b)). The thin beam 
gives us a small mass (an effective mass $m$ of $2 \mathrm{pg}$ ), while the length provides both good coupling to the microwave cavity as well as a very small spring constant of a few $\mathrm{mN} / \mathrm{m}$. The beam is placed in the cavity such that the motion of its fundamental flexural mode changes the capacitance between the cavity center conductor and the ground plane in a small section of the cavity (Fig. 2). To maximize the coupling, the gap between the beam and the ground plane is as small as is feasible (typically $1 \mu \mathrm{m}$ ), and the beam is embedded at a voltage antinode of the cavity standing wave. With the beam at this position the cavity resonance frequency shifts according to $\frac{1}{\omega_{c}} \frac{\partial \omega_{c}}{\partial x}=-\frac{\partial C_{b}}{\partial x} 4 Z_{1} \omega_{c} / 2 \pi$ for a $\lambda / 4$ cavity, where $\frac{\partial C_{b}}{\partial x}$ is the effect of the beam motion on the cavity capacitance and note $-\frac{\partial \omega_{c}}{\partial x}$ is the coupling $g$ of Eq. (1).

To detect nanomechanical motion with our microwave cavity interferometer we inject a microwave tone near a cavity resonance and monitor the phase of the transmitted signal; this phase directly reflects the cavity resonance frequency and hence the beam displacement as described above. Figure 1 shows how we extract the phase (Q) and amplitude (I) quadratures of the transmitted signal using a homodyne detection scheme.

Figure 3 a) illustrates the microwave cavity resonant response. Here we measure the relative transmission past the cavity for a set of incident microwave powers $P$ at a dilution refrigerator temperature of $17 \mathrm{mK}$, far below the $1.2 \mathrm{~K}$ critical temperature of Al. For the highest microwave powers nonlinearities become significant as the current density approaches the superconducting critical current density [16]. At low microwave power the resonant behavior is characterized by unity transmission off resonance and by a lorentzian response that dips to a value determined by the intracavity losses compared to the feedline coupling. Our imprecision in the nanomechanical beam position readout is determined by fluctuations in the associated dispersive phase signal on resonance. In Fig. 3 b) we plot our experimentally observed cavity phase fluctuations as the spectral density $S_{\phi}$. At the highest response frequencies we see a phase noise consistent with the HEMT amplifier noise, while at lower frequencies the phase noise is enhanced. This additional noise has been recently traced to two-level fluctuators in the silicon substrate [17].

An important feature of our experiment is the ability to actuate the mechanical beam without applying large magnetic fields that are incompatible with high-Q superconducting cavities [18]. In our device we incorporate a small capacitive coupling between the beam and the microwave feedline, which allows us to electrostatically drive the beam by coupling lowfrequency signals onto the feedline (Fig. 1). Using a bias-tee we introduce an ac signal near the $\omega_{m}$ and a dc voltage resulting in an electrostatic force $F_{e l}(\omega)=V_{d c} V_{a c}(\omega) \frac{\partial C_{d}}{\partial x}$ where $C_{d}$ is the drive capacitance between the feedline and the beam. To assure that the effect of the beam motion remains in the phase quadrature of the microwave signal, we design $C_{d}$ to be much smaller than $C_{b}$.

Figure 4(a) demonstrates nanomechanical displacement detection using our microwave cavity interferometer. Here the
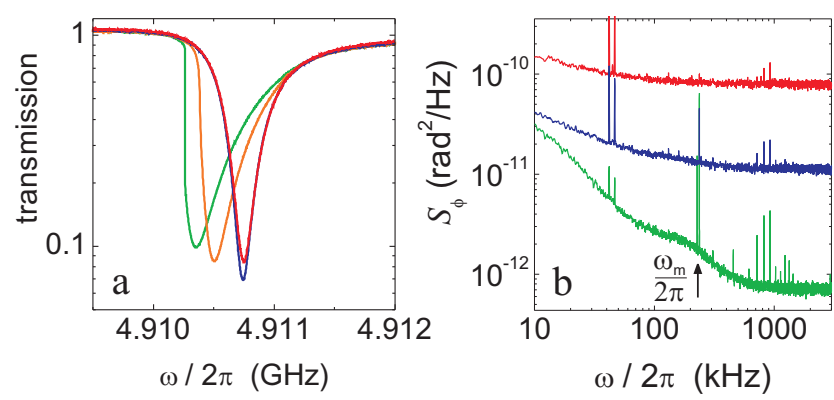

FIG. 3: (a) Power transmission past the cavity normalized to transmission off resonance. The data are shown on a logarithmic scale for incident powers of 1070 (green), 680 (orange), 68 (blue), and 11 $\mathrm{pW}$ (red). All microwave powers quoted in this work have a $3 \mathrm{~dB}$ systematic uncertainty. At the highest microwave powers the resonance becomes nonlinear and eventually bistable. At $P=68 \mathrm{pW}$, a fit to the measured response reveals an internal quality factor of $Q_{\text {int }}=38,000$, an external quality factor of $Q_{e x t}=14,000$, and a total quality factor of $Q=\left(Q_{\text {int }}^{-1}+Q_{\text {ext }}^{-1}\right)^{-1}=10,000$. (b) Measurement of the double-sideband cavity phase noise at incident powers of 1070 (green), 68 (blue), and $11 \mathrm{pW}$ (red). Motion of the mechanical beam creates the tone indicated by the black arrow; the other tones are caused by electronic interference.

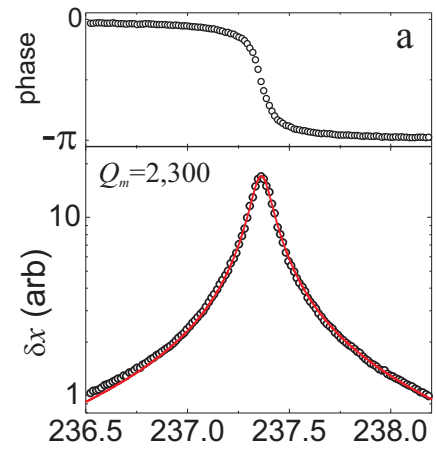

$\omega / 2 \pi(\mathrm{kHz})$

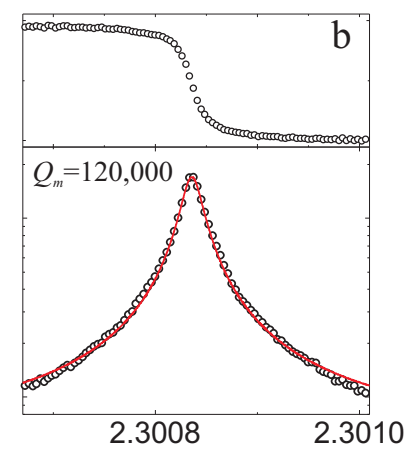

$\omega / 2 \pi(\mathrm{MHz})$
FIG. 4: (a) Resonant response of an aluminum nanomechanical beam to an electrostatic drive at $T_{\text {frig }}=17 \mathrm{mK}$. This experiment uses the beam shown in Fig. 2] b), and we find $Q_{m}=2,300$. (b) Response of a beam under tensile stress. Here the resonance is near $2 \mathrm{MHz}$ and the quality factor is greatly enhanced to $Q_{m}=120,000$. Note that as the stress is varied the change in the dissipation, as reflected by the linewidth $\gamma_{m}=\omega_{m} / Q_{m}$, is not as dramatic as the change in $Q_{m}$. The red lines are the square root of lorentzian fits to the data.

beam motion we are measuring is the response of the beam illustrated in Fig. 2(b) to an electrostatic drive. We see a clean response on a logarithmic scale, the expected $\pi$ phase shift, and good agreement with the anticipated lorentzian response (red line) of our high-Q resonance. We measure a quality factor of $Q_{m}=2,300$ and find the mechanical resonance at $\omega_{m}=2 \pi \times 240 \mathrm{kHz}$; this frequency is near our expectation for a tension-free beam with our geometry.

Figure 4(b) demonstrates the mechanical response we ob- 


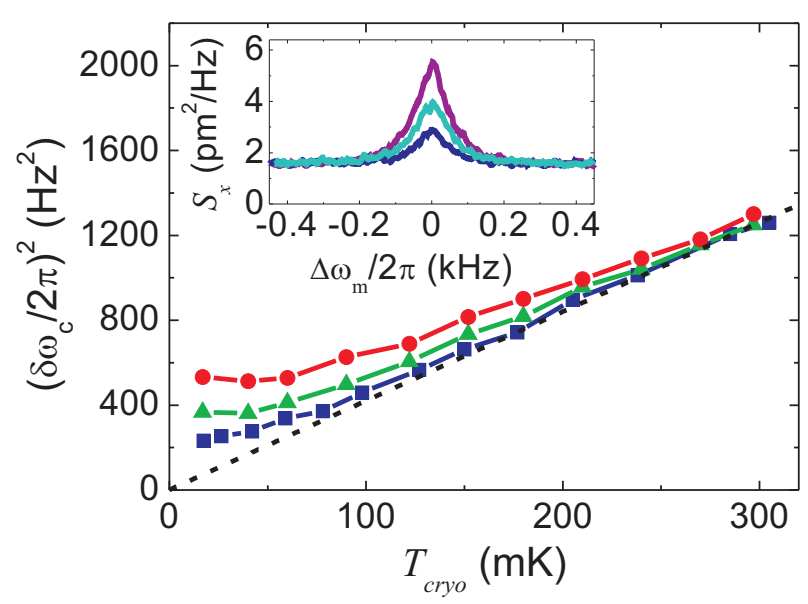

FIG. 5: Integrated mechanical beam fluctuations in units of cavity resonance frequency shift. The three data sets correspond to $P=4$ pW (blue squares), $27 \mathrm{pW}$ (green triangles), and $68 \mathrm{pW}$ (red circles). The dashed line shows the linear fit described in the text from which we extract the coupling $g$. (Inset) Lorentzian response for dilution refrigerator temperatures of $210 \mathrm{mK}, 122 \mathrm{mK}$, and $40 \mathrm{mK}$ at $P=11$ pW.

serve using a $50 \mu \mathrm{m}$ long beam fabricated from an aluminum film under tensile stress (see Methods). The stress significantly increases $\omega_{m}$ to near $2 \pi \times 2.3 \mathrm{MHz}$ and $Q_{m}$ to 120,000 . This quality factor is a surprisingly, yet pleasingly, large for a beam fabricated from an amorphous metal and of this surface to volume ratio [19, 20, 21]. When working with mechanical objects with $\omega_{m}$ in the $\mathrm{MHz}$ regime we must take into account that the sidebands generated by the beam's motion move outside the cavity bandwidth, $\gamma_{c}=\omega_{c} / Q=2 \pi \times 490$ $\mathrm{kHz}$. In this so-called good-cavity limit, to acquire the response seen in Fig. 4(b) we detune the injected microwave signal off-resonance by $\omega_{m}$ to place one sideband on the cavity resonance. Conversely if we decrease the beam tension to where $\omega_{m} \ll \gamma_{c}$ we are in the bad-cavity limit; the motion of the $240 \mathrm{kHz}$ beam is mostly in this limit, and for this case we operate with the injected microwave tone tuned to the cavity resonance.

By measuring the non-driven, thermomechanical motion of the beam, we can characterize the sensitivity and backaction of the microwave measurement. The expected thermally driven displacement fluctuations of our high-Q mechanical resonators at a bath temperature $T$ is given by

$$
S_{x}\left(\Delta \omega_{m}\right)=\frac{1}{\left(m \omega_{m} \gamma_{m}\right)^{2}} \frac{4 m \gamma_{m} k_{b} T}{1+4 \Delta \omega_{m}^{2} / \gamma_{m}^{2}}
$$

where $\Delta \omega_{m}=\omega-\omega_{m}$. The inset to Fig. 5 shows nondriven response at three different values of the dilution refrigerator temperature $T_{\text {frig }}$. (Here and in the remainder of our experimental results we will be studying the $240 \mathrm{kHz}$ mechanical beam discussed above.) The white noise background is the imprecision $S_{x}^{i m}$, while the height of the peak above the background describes the real fluctuations in the beam position. To understand the temperature-dependent response (and to calibrate the beam to cavity coupling) we can examine the integrated signal under the lorentzian as a function of $T_{\text {frig }}$. Figure 5 shows the integrated mechanical motion in the experimental units of cavity resonance frequency shift $\delta \omega_{c}$ for a set of incident microwave powers $P$ (see Methods).

In an ideal system, the integrated response should depend linearly on the temperature according to $\delta \omega_{c}^{2}=\frac{g^{2} k_{b}}{m \omega_{m}^{2}}\left(T_{\text {frig }}+\right.$ $\left.T_{b a}\right)$ where $T_{b a}=S_{F}^{b a} / 4 k_{b} m \gamma_{m}$ is the equivalent backaction temperature. If we focus on lowest microwave power results (blue squares) and the highest dilution refrigerator temperatures we see that the response is linear down to $\sim 100$ $\mathrm{mK}$. Here linear fits reveal that the backaction is small compared to relevant uncertainties, and we extract a coupling of $g=2 \pi \times 1.16 \mathrm{kHz} / \mathrm{nm}$ using points above $127 \mathrm{mK}$ (dashed line). This value of $g$ corresponds to a capacitance change of $\frac{\partial C_{b}}{\partial x}=170 \mathrm{aF} / \mu \mathrm{m}$, which is consistent with our numerically calculated expectation.

At lower values of $T_{\text {frig }}$ and higher microwave powers (green triangles and red circles) the beam temperature decouples from $T_{\text {frig }}$ leading to a saturation behavior. The microwave power dependence suggests that the additional fluctuations are related to microwave power dissipation. However, by using a different cavity on the same chip as a crude thermometer, we know that the dissipated power does not heat the entire chip above $T_{\text {frig }}$. The heating of the beam by the microwave power must be a more local effect.

Given the nonlinear dependence of the beam fluctuations on $T_{\text {frig }}$ we must be cautious in how we define and extract $T_{b a}$ for our measurement. We believe an honest metric is the equivalent temperature of the beam fluctuations at our base temperature $\left(T_{\text {frig }}=17 \mathrm{mK}\right)$, which we will refer to as the saturation temperature $T_{\text {sat }}$. This temperature is a conservative upper limit on our ability to measure a backaction force since extrapolating $T_{b a}$ from only the highest $T$ points would yield a smaller value. In Fig. 6 we plot $T_{\text {sat }}$ along with the imprecision temperature $T_{i m}=S_{x}^{i m} m \omega_{m}^{2} \gamma_{m} / 4 k_{b}$ as a function of $P$. At the lowest powers the imprecision dominates over the beam fluctuations. As the power is increased the imprecision drops linearly with power as expected, but at the highest microwave powers it is enhanced by cavity phase noise to a value above our microwave amplifier noise floor (dashed line).

From the results in Fig. 6 we can assess how close an approach we have made to the SQL. The minimum uncertainty in continuous position detection subject to the Heisenberg constraint $S_{x}^{i m} S_{F}^{b a}=\hbar^{2}$ occurs at the point where $S_{x}^{i m}=S_{x}^{b a}=S_{F}^{b a} /\left(m \omega_{m} \gamma_{m}\right)^{2}$. Here the imprecision and the backaction both contribute $S_{x}(S Q L)=\hbar / m \omega_{m} \gamma_{m}$. We can convert the imprecision and saturation temperatures in Fig. 6 into a position spectral density compared to this minimum value via $S_{x} / S_{x}(S Q L)=4 k_{b} T / \hbar \omega_{m}$. This result is shown on the right axis of Fig. 6. Our limit on the imprecision alone at the highest microwave powers corresponds to, in linear units, $30 \sqrt{S_{x}(S Q L)}$. The total minimum position 


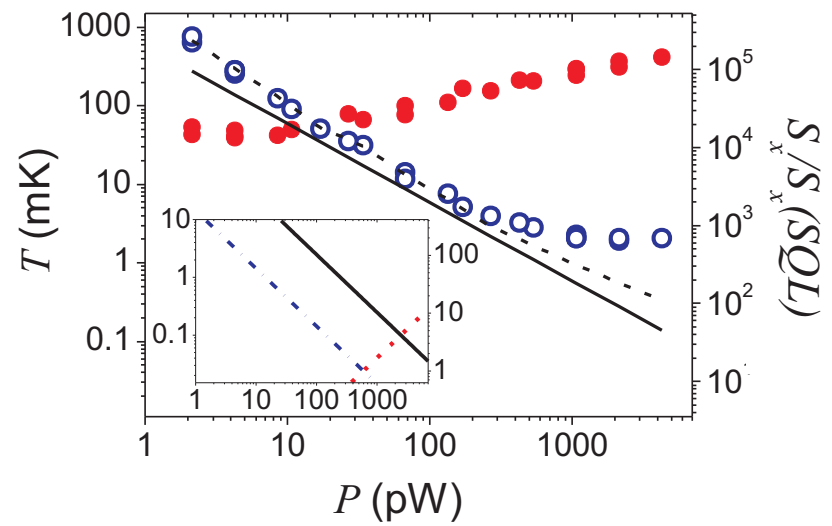

FIG. 6: Imprecision temperature (blue open circles) and saturation temperature (red circles) as a function of incident microwave power. The lines represent the expected imprecision due to our microwave amplifier; the dashed line includes the loss of microwave power in the cavity, while the solid line represents the ideal expectation in the limit of a lossless cavity. On the right vertical axis we display the position uncertainty in units of $S_{x}(S Q L)$. (Inset) Over the same range of power we calculate the quantum-limit of displacement detection for optimized parameters. We consider the ideal case of a lossless onesided cavity coupled to one port using the parameters quoted in the text. The lines correspond to the shot-noise limit (dash-dot line), the quantum backaction (dotted line), and the imprecision due to a $T_{N}=5 \mathrm{~K}$ voltage amplifier (solid line).

uncertainty we achieve occurs at $P=20 \mathrm{pW}$ and is given by $\sqrt{S_{x}^{t o t}}=130 \sqrt{S_{x}^{t o t}(S Q L)}$, where to be explicit $S_{x}^{t o t}$ here is $S_{x}^{i m}+S_{x}^{s a t}$, which is an upper bound on our ability to measure $S_{x}^{i m}+S_{x}^{b a}$.

We can also extract absolute values for the achieved sensitivity. Our imprecision is limited to $200 \mathrm{fm} / \sqrt{\mathrm{Hz}}$ at the highest microwave powers, which is a modest achievement compared to optical systems [2]. On the other hand our total force sensitivity, $\sqrt{S_{F}^{t o t}}=\sqrt{4 k_{b}\left(T_{i m}+T_{s a t}\right) m \gamma_{m}}$, is $3 \mathrm{aN} / \sqrt{\mathrm{Hz}}$ at $P=20 \mathrm{pW}$. This value is near the record mechanical force sensitivity of $0.8 \mathrm{aN} / \sqrt{\mathrm{Hz}}$ achieved using a fiberoptic interferometer and a silicon cantilever [22].

To closer approach the SQL with our microwave cavity interferometer the foremost task will be to decrease the dissipation that likely leads to our observation of a finite $T_{\text {sat }}$; this dissipated power is determined by $Q_{\text {int }}$ compared to $Q_{e x t}$ and for our current work is $2 \mathrm{pW}$ at $P=20 \mathrm{pW}$. To decrease the dissipated power the nanomechanical beam processing must be developed to be compatible with a very large $Q_{\text {int }}$. Another route to improvement is to increase $\omega_{m}$ which will decrease thermal fluctuations and the dissipative force compared to quantum fluctuations, as well as decrease the $\nu^{-1 / 2}$ cavity phase noise. However, to maintain the force sensitivity, an increase in the mechanical spring constant should be accompanied by an increase in the beam to cavity coupling $g$. One option for increasing $g$ would be to decrease the total cavity capacitance by operating at larger $\omega_{c}$ or by utilizing a higherimpedance microwave cavity or lumped-element circuit.
It is instructive to assess what we could achieve in future experiments utilizing optimized, yet likely realizable, parameters. Consider a device described by $\omega_{m}=2 \pi \times 2$ $\mathrm{MHz}, m=2 \mathrm{pg}, Q_{m}=100,000, \omega_{c}=2 \pi \times 12 \mathrm{GHz}$, $Q_{\text {int }} \gg Q_{\text {ext }}=3,000$, and $g=2 \pi \times 20 \mathrm{kHz} / \mathrm{nm}$. Further, assume we modify our geometry to measure in reflection off of a single-sided cavity with a single port. For this more ideal geometry and a microwave probe at $\omega_{c}$, the quantum-limited imprecision expected for detection via a classic square-law detector would be the shot-noise limit

$$
S_{x}^{s n}=\frac{\hbar \omega_{c}\left(1+4\left(\omega_{m} / \gamma_{c}\right)^{2}\right)}{2\left(g / \omega_{c}\right)^{2} P(4 Q)^{2}},
$$

and correspondingly $S_{F}^{b a}=\hbar^{2} / S_{x}^{s n}$. Our calculated expectation for $S_{x}^{s n}$ and $S_{F}^{b a}$ is shown in the inset to Fig. 6 We also include the noise of a possible HEMT amplifier (solid line). This level could be improved by incorporating better microwave amplifiers that could soon be available given recent interest in developing novel microwave amplifiers near the quantum limit [23, 24, 25]. However, even a quantum-limited voltage amplifier measuring both field quadratures will result in an imprecision a factor of two above the shot-noise limit [26], and to reach the SQL one must utilize an amplifier that detects only one quadrature.

Overall the results of the calculations in the inset to Fig. 6 are promising; even with our current HEMT amplifier the minimum uncertainty (assuming ideal backaction) would be a factor of $\sim 2.0$ above the quantum limit in linear units. While the equivalent backaction temperatures that must be measured are only a fraction of a $\mathrm{mK}$, with precise measurements it is in principle possible to extract a $T_{b a}$ that is much smaller than the bath temperature. Most importantly, the shot-noise limit and quantum backaction intersect at an achievable incident microwave power of $600 \mathrm{pW}$.

The novel coupled mechanical and microwave system we have demonstrated is not only promising as a detector, but also could be adapted to cool nanomechanical resonators towards their ground state. As first explored by Braginsky [27] dynamical backaction due to radiation pressure can lead to a passive cooling or heating of the mechanical motion when the injected tone is detuned from the cavity resonance. This effect has recently been observed in optical cavities with micromechanical mirrors [5, 6, 7, 8, 9] and radiofrequency circuits [28], and it has been suggested as a method of cooling and manipulating a beam coupled to a transmission-line cavity [29, 30]. Especially interesting is the possibility of passive cooling utilizing our ability to access the good-cavity limit, as it is in this limit that one can in principle cool fully to the mechanical ground state [31, 32]. In addition to passive cooling, our ability to apply electrostatic forces while reading out displacement with our microwave cavity interferometer makes us well-poised to implement feedback cooling of our nanomechanical beams [33, 34]. 


\section{METHODS}

The device is fabricated using a combination of electronbeam lithography and photolithography, and the beam and cavity are formed from the same thermally evaporated aluminum film in a single liftoff process. The beam is patterned directly on the silicon substrate and suspended at the end of the process with an isotropic, dry silicon etch. A relatively deep etch of $4 \mu \mathrm{m}$ is typically required to release our mechanical beams with low spring constants. An insulating layer of $\mathrm{SiO}_{2}$ underneath the rest of the pattern is used to protect the coplanar waveguide slots during the etch.

Our initial thermally evaporated aluminum film contains significant compressive stress. To adjust the stress of the aluminum beam we partially anneal the device at $150-350{ }^{\circ} \mathrm{C}$ in atmosphere before releasing the beam from the substrate. The final stress of the beam at cryogenic temperatures is affected by the differential thermal coefficient of expansion of silicon and amorphous aluminum. We estimate that between room and cryogenic temperatures the aluminum film shrinks by a few tenths of a percent compared to its clamping locations. Hence, the beam of Fig. (4)a) has significant compressive stress at room temperature (see Fig. 2(b)) but less compressive stress at $T_{\text {frig }}$; the beam of Fig. 4 (b) has little stress at room temperature but significant tensile stress at $T_{\text {frig }}$.

Since our IQ mixer (Marki IQ03076XP) has orthogonal outputs near $5 \mathrm{GHz}$, we can place all of the phase information in the $\mathrm{Q}$ channel by rotating the phase of the signal into the LO of the mixer. The voltage fluctuations measured in the Q quadrature $S_{V}^{Q}$ are then all that is required to extract the integrated cavity resonance frequency shift plotted in Fig. 5. The relationship between the cavity resonance frequency fluctuations and the voltage fluctuations is

$$
S_{\omega_{c}}=\frac{\omega_{c}^{2}\left(1+4\left(\omega_{m} / \gamma_{c}\right)^{2}\right)}{(2 Q)^{2} V_{0}^{2}\left(1-S_{\min }\right)^{2}} S_{V}^{Q}
$$

where $V_{0}$ is voltage amplitude of the transmission off resonance and $S_{\min }$ is the normalized transmission past the cavity on resonance. The term $1+4\left(\omega_{m} / \gamma_{c}\right)^{2}$ accounts for filtering of the cavity response at $\omega_{m}$ and becomes 1 in the bad-cavity limit. The integrated response in units of cavity resonance frequency shift is then given by $\delta \omega_{c}^{2}=S_{\omega_{c}}^{0} \gamma_{m} / 4$, where $S_{\omega_{c}}^{0}$ is the magnitude of the lorentzian response at $\Delta \omega_{m}=0$. The $T$ dependence of the $Q, S_{\text {min }}$, and $Q_{m}$ must be taken into account in these conversions, but we restrict our measurements to below $300 \mathrm{mK}$ where the values change by $<20 \%$.

In Fig. 6 we extend our measurement into the regime where the cavity resonance becomes nonlinear (see Fig. 3) and hence conversion between $S_{V}^{Q}$ and $S_{\omega_{c}}^{Q}$ becomes less straightforward to calculate. To extract this conversion at nonlinear microwave powers, we perform a separate calibration experiment in which we apply a constant electrostatic drive and compare the beam response at high microwave power to the known response at low power.

\section{ACKNOWLEDGEMENTS}

We acknowledge support from the NSF Physics Frontier Center for AMO Physics and from the National Institute of Standards and Technology. C. A. R. acknowledges support from the Fannie and John Hertz Foundation. We thank R. J. Schoelkopf, S. M. Girvin, K. D. Irwin, D. Alchenberger, M. A. Castellanos-Beltran, and N. E. Flowers-Jacobs for enlightening conversations and technical assistance.

* Current address: Norman Bridge Laboratory of Physics 12-33, California Institute of Technology, Pasadena, California 91125

$\dagger$ E-mail: konrad.lehnert@jila.colorado.edu

[1] C. M. Caves, Quantum-mechanical noise in an interferometer, Phys. Rev. D 23, 1693 (1981).

[2] O. Arcizet, P.-F. Cohadon, T. Briant, M. Pinard, A. Heidmann, J.-M. Mackowski, C. Michel, L. Pinard, O. Français, and L. Rousseau, High-sensitivity optial monitoring of a micromechanical resonator with a quantum-limited optomechanical sensor, Phys. Rev. Lett. 97, 133601 (2006).

[3] I. Tittonen, G. Breitenbach, T. Kalkbrenner, T. Müller, R. Conradt, S. Schiller, E. Steinsland, N. Blanc, and N. F. de Rooij, Interferometric measurements of the position of a macroscopic body: Towards observation of quantum limits, Phys. Rev. A 59, 1038 (1999).

[4] V. B. Braginsky, in Systems with small dissipation, edited by K. S. Thorne and C. Eller (University of Chicago Press, Chicago, 1985).

[5] O. Arcizet, P.-F. Cohadon, T. Briant, M. Pinard, and A. Heidmann, Radiation-pressure cooling and optomechanical instability of a micromirror, Nature 444, 71 (2006).

[6] S. Gigan, J. R. Böhm, M. Paternostro, F. Blaser, G. Langer, J. B. Hertzberg, K. C. Schwab, D. Bäuerle, M. Aspelmeyer, and A. Zeilinger, Self-cooling of a micromirror by radiation pressure, Nature 444, 67 (2006).

[7] D. Kleckner and D. Bouwmeester, Sub-kelvin optical cooling of a micromechanical resonator, Nature 444, 75 (2006).

[8] A. Schliesser, P. Del'Hayer, N. Nooshi, K. J. Vahala, and T. J. Kippenberg, Radiation pressure cooling of a micromechanical oscillator using dynamical backaction, Phys. Rev. Lett. 97, 243905 (2006).

[9] J. D. Thompson, B. M. Zwickl, A. M. Jayich, F. Marquardt, S. M. Girvin, and J. G. E. Harris, Strong dispersive coupling of a high finesse cavity to a micromechanical membrane, Nature 452, 72 (2008).

[10] M. D. LeHaye, O. Buu, B. Camarota, and K. C. Schwab, Approaching the quantum limit of a nanomechanical resonator, Science 304, 74 (2004).

[11] A. Naik, O. Buu, M. D. LaHaye, A. D. Armour, A. A. Clerk, M. P. Blencowe, and K. C. Schwab, Cooling a nanomechanical resonator with quantum back-action, Nature 443, 193 (2006).

[12] N. E. Flowers-Jacobs, D. R. Schmidt, and K. W. Lehnert, Intrinsic noise properties of atomic point contact displacement detectors, Phys. Rev. Lett. 98, 096804 (2007).

[13] P. K. Day, H. G. LeDuc, B. A. Mazin, A. Vayonakis, and J. Zmuidzinas, A broadband superconducting detector suitable for use in large arrays, Nature 45, 817 (2003).

[14] A. Wallraff, D. Schuster, A. Blais, L. Frunzio, R.-S. Huang, J. Majer, S. Kumar, S. M. Girvin, and R. J. Schoelkopf, Circuit 
quantum electrodynamics: Coherent coupling of a single photon to a Cooper pair box, Nature 431, 162 (2004).

[15] J. A. B. Mates, G. C. Hilton, K. D. Irwin, and L. R. Vale, Demonstration of a multiplexer of dissipationless superconducting quantum interference devices, Appl. Phys. Lett. 92, 023514 (2008).

[16] T. Dahm and D. J. Scalapino, Theory of intermodulation in a superconducting microstrip resonator, J. Appl. Phys. 81, 2002 (1997).

[17] J. Gao, J. Zmuidzinas, B. A. Mazin, H. G. LeDuc, and P. K. Day, Noise properties of superconducting lithographed microwave resonators, Appl. Phys. Lett. 90, 102507 (2007).

[18] L. Frunzio, A. Wallraff, D. Schuster, J. Majer, and R. Schoelkopf, Fabrication and characterization of superconducting circuit QED devices for quantum computation, IEEE Trans. Appl. Supercond. 15, 860 (2005).

[19] K. L. Ekinci and M. L. Roukes, Nanoelectromechanical systems, Rev. Sci. Instrum. 76, 061101 (2005).

[20] S. S. Verbridge, J. M. Parpia, R. B. Reichenbach, L. M. Bellan, and H. G. Craighead, High quality factor resonance at room temperature with nanostrings under high tensile stress, J. Appl. Phys. 99, 124304 (2006).

[21] B. M. Zwickl, W. E. Shanks, A. M. Jayich, C. Yang, A. C. Bleszynski, J. D. Thompson, and J. G. E. Harris, High quality mechanical and optical properties of commercial silicon nitride membranes, ArXiv: 0711.2263 (2007).

[22] H. J. Mamin and D. Rugar, Sub-attonewton force detection at millikelvin temperatures, Appl. Phys. Lett. 79, 3358 (2001).

[23] B. Yurke, L. R. Corruccini, P. G. Kaminsky, L. W. Rupp, A. D. Smith, A. H. Silver, R. W. Simon, and E. A. Whittaker, Observation of parametric amplification and deamplification in a Josephson parametric amplifier, Phys. Rev. A 39, 2519 (1989).

[24] M. Muck, J. B. Kycia, and J. Clarke, Superconducting quantum interference device as a near-quantum-limited amplifier at 0.5
GHz, Appl. Phys. Lett. 78, 967 (2001).

[25] M. A. Castellanos-Beltran and K. W. Lehnert, Widely tunable parametric amplifier based on a superconducting quantum interference device array resonator, Appl. Phys. Lett. 91, 083509 (2007).

[26] C. M. Caves, Quantum limits on noise in linear amplifiers, Phys. Rev. D 26, 1817 (1982).

[27] V. B. Braginsky and A. B. Manukin, in Measurement of weak forces in physics experiments, edited by D. H. Douglass (The University of Chicago Press, Chicago, 1977).

[28] K. R. Brown, J. Britton, R. J. Epstein, J. Chiaverini, D. Leibfried, and D. J. Wineland, Passive cooling of a micromechanical oscillator with a resonant electric circuit, Phys. Rev. Lett. 99, 137205 (2007).

[29] F. Xue, Y. D. Wang, Y. Liu, and F. Nori, Cooling a micromechanical beam by coupling it to a transmission line, Phys. Rev. B 76, 205302 (2007).

[30] D. Vitali, P. Tombesi, M. J. Woolley, A. C. Doherty, and G. J. Milburn, Entangling a nanomechanical resonator and a superconducting microwave cavity, Phys. Rev. A 76, 042336 (2007).

[31] F. Marquardt, J. P. Chen, A. A. Clerk, and S. M. Girvin, Quantum theory of cavity-assisted sideband cooling of mechanical motion, Phys. Rev. Lett. 99, 093902 (2007).

[32] I. Wilson-Rae, N. Nooshi, W. Zwerger, and T. J. Kippenberg, Theory of ground state cooling of a mechanical oscillator using dynamical backaction, Phys. Rev. Lett. 99, 093901 (2007).

[33] M. Poggio, C. L. Degen, H. J. Mamin, and D. Rugar, Feedback cooling of a cantilever's fundamental mode below $5 \mathrm{mK}$, Phys. Rev. Lett. 99, 017201 (2007).

[34] C. Genes, D. Vitali, P. Tombesi, S. Gigan, and M. Aspelmeyer, Ground-state cooling of a micromechanical oscillator: Comparing cold-damping and cavity-assisted cooling schemes, arXiv:0705.1728v4 (2008). 